\title{
CHANGE MANAGEMENT WITH THE AID OF A GENERIC MODEL FOR RESTRUCTURING BUSINESS PROCESSES
}

\author{
Vedenik, G. ${ }^{*} \&$ Leber, M.* \\ ${ }^{*}$ Veplas RTM d.o.o., Cesta Simona Blatnika 11, 3320 Velenje, Slovenia \\ ${ }^{* *}$ University of Maribor, Faculty of Mechanical Engineering, Smetanova 17, 2000 Maribor, Slovenia \\ E-Mail: gregor.vedenik@veplas.si
}

\begin{abstract}
In the last few decades, the Slovenian business environment has experienced a number of transitions, market, political and financial changes. Companies, which failed to incorporate these changes into their strategies, quickly fell into difficulties or went into liquidation. Managing change is of key importance if companies or organisations want to remain competitive. As a means of support companies can employ various approaches, techniques, tools and models; these, however, are not always adapted to the needs - deriving from the strategy and the environment - or the type of the company, especially if these are small or medium-sized enterprises. This article presents the concept of a generic model for managing changes. Based on simulation, this model will support the management of small and medium-sized enterprises in detecting problems, defining solutions and implementing necessary changes in business processes. With the developed model a simulation of changes in balance sheet data for sales and the profit achieved was carried out, the goal of increasing sales defined and the limits set. The model offered seven opportunities which have all been commented on appropriately. By utilising this model, a static company changes into a dynamic one capable of quick restructuring whenever necessary.

(Received in August 2014, accepted in May 2015. This paper was with the authors 4 months for 2 revisions.)
\end{abstract}

Key Words: Change Management Model, Business Processes, Life Cycle, Pathological Problems, Simulation

\section{INTRODUCTION}

We live in a world that is full of changes. Living with changes and managing them is a key competency if we want to develop and grow, and be able to take advantage of new challenges, as Charles Darwin taught in his evolution theory. Changes are unpredictable, but they are also commonplace and ever-present. Everyone faces changes as they pass through various stages of life. Our attitude to change dictates how we view them; they can be either an opportunity or a problem. A change requires us to take different decisions and to behave differently because we are faced with a new situation. Every problem and every opportunity that accompanies a change requires solutions which may lead to new changes and new situations, which again bring about new problems and opportunities. This translates to a recurrent cycle with constantly occurring changes causing constant opportunities and problems.

In the last three decades, Slovenia has faced great changes (changes in the political system, creating an independent country, becoming a member of NATO and the EU, adopting two new currencies, transition of companies from state-owned to privately owned, etc.) which have transformed the image of the country and its citizens. The emergence of the economic and financial crisis has been a cold shower and forced many once large and promising companies to ingloriously close their doors. It only takes a look at the list of companies recognised by the Chamber of Commerce and Industry of Slovenia, or those which were awarded the Gazela award for fastest growing companies twenty or thirty years ago, to see that today they no longer exist or are in insolvency procedures [1]. It is impossible to say that the companies were not aware of growth models or theoretical assumptions arising therefrom; however, it is realistic to state that most of them disregarded the assumptions. Equally, it can 
be argued that in these ruined companies success managed the company and not vice versa. Furthermore, companies set foot in other sectors and industries without being precisely aware of how that sector or industry functioned, or lacking the proper skills and knowledge to operate in a certain field. Companies were neither acquainted with nor did they solve pathological problems and as a consequence many of them went into liquidation. The following is a summary of the most typical mistakes made by companies during the transition period:

- unawareness or ignorance of the status quo in an organisation or the environment;

- thinking that changes are not necessary or that they can wait;

- lack of competencies and resources for simultaneously introducing changes;

- the entire change management process was too complex and not easy to use;

- overall late response to changes in an organisation or the environment;

- lack of financial recourses.

Based on the findings it can be said that the Slovenian business environment is insusceptible to urgent changes or is unable to manage them. This article uses the established starting points to create and develop a generic model for change management, useful particularly in small and medium-sized enterprises to assist the management in detecting problems, defining solutions and implementing urgent changes.

\section{CHANGE MANAGEMENT AND THE ORGANISATION'S STRATEGY}

\subsection{Change management as a mean of an organisation's transition to an optimal state}

How to implement changes is a key question for every organisation or company. Change management (also known as change control) is a professional discipline which focuses on supporting organisations on their way to a successful transition from a less-than-ideal status quo to a desired future state. Change management is one of the skills every manager should master to a sufficient degree because it represents an integral part of business operations and the process of constant change. It denotes a dispersed set of processes, tools, techniques, methods and approaches for achieving a desired state through change.

Change management approaches have two main objectives:

- to assist the organisation in achieving its goals which cannot be attained with the existing organisational structure, functioning and client servicing, and

- to minimise the adverse effects of any changes made.

Change management incorporates the meticulous planning of processes which lead to successful change implementation and makes it possible to create a better future. Needless to say, these processes can be strongly influenced by our willingness to adopt changes and the frequency of such processes. Huczynski and Buchanan (1985) define learning as a process of acquiring knowledge through experience, resulting in behavioural changes [2].

Change management stands for and includes well-thought-out planning and implementing, as well as the involvement of and consultation with people influenced by the changes. Imposing changes - no matter how urgent they may be - can be met by a negative reaction from employees. Therefore it is necessary to make the changes as clear as possible and include those affected by them in the change-making process. Employees are not always fond of changes but they do understand that for an organisation to grow alterations are inevitable. It is very difficult for them to adapt to a change unless the management processes of a company are altered and brought in line with the change as well. It is essential that the change is attainable, realistic and measurable. Regardless of the organisational level at which the change is introduced, it is always humans with their characteristics who accept or reject it. 
Thus it depends on them whether a change will be successful or not [3]. Technology, civilisations and creative thinking pave the way for future changes as well. It can be said that the implementation of changes has become a routine [4].

An organisation must be proactive, stay ahead of the competition and as such lead the game. Changes in an organisation occur in response to business and economic events, or as an answer or reaction to a certain incentive. It is therefore necessary to understand why and how changes occur, and what has to be done to turn the implementation of changes into a successful process. To implement the most important changes it is relevant to stay focused on the triggers of change.

\subsection{Organisation's strategy development and implementation}

It is essential not to ignore changes because, sooner or later, an organisation will have to face them. For an organisation or company to survive and prosper strategies which realistically reflect its capability to implement various future scenarios must be adopted. The implementation of changes must become a continuous process - with various causes and needs - so as to facilitate the introduction of changes which can be planned. Whether a company is competitive or not depends on its ability to transform its key business processes into strategic capabilities used to continuously deliver better customer value than the competition.

The research has shown that while on the one hand many companies have very detailed strategies, on the other hand some could not even answer a simple yet very important question in terms of the definition of a company's or organisation's strategy: What is the mission of our company (organisation) and why do customers need us? Based on this answer a concept of a new and desired future form of organisation (vision) can be devised.

Answers to the following questions can assist in defining an organisation's purpose:

- What will be the company's basic area of operation (products, services)?

- Which market niches exist and what are the possibilities for our company to fill those niches?

- What is the level of quality and trust (reliability, timeliness) that we achieve/will achieve?

- How able are we to provide competitive prices?

- How adaptable are we (taking measures in times of market fluctuations, changing products)?

- How wide is the range of our business activities?

- Who are the competitors?

- What are our basic values and beliefs?

- What is our business philosophy?

- Where do you see the organisation's social responsibility?

- What is the public image of the company? [5]

\subsection{Organisation's life cycle in addressing changes}

An essential element of every strategy represents the future growth and development of an organisation or company, which must be strategically planned and controlled. If growth is too fast and surpasses the company's basic capabilities in terms of human, financial, technological and other resources, the discrepancy can lead to the company's failure. In the design of the generic model for change management created in the framework of this study, basic assumptions related to growth and the company's growth management were taken into account. To explain how firms grow, theoretical models are used - based on the characteristics of a company's development life cycle, or the dynamic growth process over various life cycle phases - but practical models based on the life cycle are also utilised. 
Growing companies are characterised by business models grouped in the following three categories:

- Companies which are successful at offering high-quality services or products, whether they develop them on their own or acquire from others. Usually their growth is in line with the economic conjuncture.

- Companies which meet specific customers' needs.

- Companies with a mind-set that is primarily focused on creating needs of which the customers are not yet aware [6].

When a company is moving from one life cycle phase to another it must break from old behaviour patterns and adopt new ones as fast as possible. In the transition from an old to a new behaviour pattern problems occur which can be considered "normal". It is not possible to avoid these normal problems because when new ways are being introduced the organisation must learn, develop its skills and set aside a part of its capacities for this purpose. But if an organisation is investing energy to overcome difficulties which hinder changes, then these are pathological problems which must be dealt with very seriously because they differ from the normal problems in their intensity and significance, and can be labelled as chronic. If pathological problems (e.g. uncontrolled negative cash flow, constant outflow of key employees, unsolved problems related to quality, rapid decline in market share, etc.) are not addressed promptly they may endanger the organisation's capability to survive. Organisations dealing with such problems cannot afford a therapy, because a therapy takes time which they do not have. Instead of a therapy they must shift the entire organisation [7]. A company must endeavour to create a state in which the function, form, flexibility and self-control are consistent. As a result it can change in a controlled manner, achieve optimal results and maintain such an effect in the long term. It is even better if a company is able to develop such capabilities simultaneously.

To be able to make the right decisions relating to an organisation's successful transition from one life cycle phase to another, a very good understanding of the causes which lead to growth and ageing of an organisation is needed [8].

\subsection{Changes and management}

A change may mean that the reasons for the existence of an organisation or its basic strategies are changed, or only minor revisions or small activities are carried out. A change can immediately affect the entire organisation or be concentrated only on a certain segment in the organisation, such as an individual team. Typically, organisations at all times address several changes in parallel. In this context, management in implementing changes is crucial. The role of the organisation's management is to carry out necessary changes which bring about new problems, to reintegrate the organisation to solve these problems, and to prepare the organisation for new changes and problems. So-called change managers lead the change process and reintegrate the system into a new whole. A change manager is a person responsible for the successful implementation of a change and his role is similar to a project manager. A change manager must identify him-/herself with the change and set an example to other employees. With his/her skills a change manager is obliged to assist other employees in working with the change because not one person possesses exactly the same skills, capabilities, emotions and desires as another, or has the same opinion and experience. A change manager must be able to correctly predict and understand how the employees will react to the change; otherwise there is a strong likelihood that the employees will reject it. Cameron and Green [3] talk about a management model which functions as a strong reminder for the managers whose task it is to regulate three key dimensions (results, interests, emotions). Change managers must divide their efforts between all three dimensions of an organisational change. 
To reduce the risk for an organisation, change management must find a way to introduce or implement changes efficiently and manage them skilfully throughout all process phases [9]. High-risk decision-making processes should always be supported by the experts' opinion with realistic predictions to help the managers and to assure positive effects and sustainable competitive advantage $[10,11]$. This can only be achieved by a systematic approach and the introduction of a model for managing changes comprehensively at all levels.

\section{CORNERSTONES OF A CHANGE MANAGEMENT MODEL}

\subsection{Observance of fundamental principles in designing a change management model}

In the design of the model certain logical sequences must be taken into account; that is to say, the cornerstones of the model, which could also be described as the sub-models of the superordinate generic process model defined. Furthermore, it is essential to understand a number of important theoretical starting points in relation to the nature of changes, and a set of questions and possibilities which must be addressed when a change management approach is being developed and the model designed.

A review of literature shows a number of different change models, where each of them has its own characteristics and is more suitable for a certain type of organisation $[3,4,12-$ 17]. Some models are useful when change processes are analysed at the beginning of the initiative. Conducting analyses of sections and discussions on the current and the final state are very useful tools as well. Other models are more suitable for solving isolated and less complex matters. At the same time they are not appropriate for too simple organisational changes because they disregard resistance and overlook the interdependence between business units and subsystems.

In the framework of different studies various principles in designing a change management model were analysed. It makes sense to take these principles into account in the design and construction of a useful generic model which can be used to carry out any kind of simulation associated with the phenomenon of changing events:

- The start-up of implementing changes can be problematic, especially in organisations in which changes are not frequent or are carried out to a limited extent. In such cases models which put great emphasis on the proper implementation of the initial steps - the construction of a coalition and setting up a vision - are very useful.

- One of the important factors which have to be examined and taken into account is the simplicity and efficiency of a model. Thus a model can be used at each point in the change process.

- The model utilised should also provide a check-list for the change process. Such systems are very useful for conducting analyses in the period following the change, which are often used to establish what went wrong.

- Certain changes cannot be avoided. In such cases it is useful to apply models which tackle inevitable changes, such as labour force surpluses, mergers, fusions. Models of this kind are specific and are less efficient if changes are not inevitable.

- In addition there are models which combine a larger number of key elements in organisational change in a network process and produce very useful check-lists.

- Certain models give a greater dose of realism. The use of these models is somewhat limited at times when business events happen dynamically and rapid results as well as maximal efficiency are required.

- By combining various approaches certain models may lose their value. Usually they require a definition of the roles which can be difficult at times when it comes to complex solutions. 


\subsection{Fundamental cornerstones of designing a generic change management model}

The main purpose of the study was to define cornerstones for specific company types. We selected a medium-sized organisation, active in the Central European transition business environment. Based on the studies performed and the limits set, the fundamental cornerstones of a generic change management model were defined:

a. In order for a change to be successful it is of key importance to transform the recognised need for change into a desire for change and define who will manage the change and build interrelations in an organisation.

b. The generic process model is primarily meant for a specific group of users; therefore it has to derive from an overview of the status quo and identify the desired future state.

c. Every change strategy includes questions connected to people who go through the change process. The success or otherwise of the change depends on them.

d. A change manager must recognise the need or be aware of the needs of the people within and outside of the organisation and identify whether they can sabotage the change or contribute to the change process. A motivated change team is needed for implementation.

e. The reasons for resisting the change must be identified, because the general commitment level can influence the change support level.

f. The purpose of the change initiative is to move the organisation to a state in which goals can be realised, or to a transition between the status quo and the desired state. The gap between these two states must be defined and filled.

g. In order for the goal to be attainable, it has to be supported by the most appropriate success or effects measurement. If the goals of changes are clearly defined, the effects will be quite visible as well.

h. Change is a complex activity and it must be controlled by a wide-reaching, well-organised and flexible plan, although in reality things may go differently.

i. While the change project is progressing the stage of the change that is frequently the most risky is reached; that is, implementing the change as a whole or parts of it.

j. Implementing the change within an organisation is a step in the change process that includes measures for change realisation.

k. Training and continual improvements must become an integral part of the change process.

1. When building the model the development of lean thinking has to be considered or the various techniques and tools of lean operating systems must be studied.

$\mathrm{m}$. Change monitoring enables progress to be monitored according to the plan and according to the goals achieved; this enables the adoption of measures that depend on individual situations.

\section{CHANGE MANAGEMENT MODEL}

The change management model is planned in a way that enables a simulation while considering theoretical presumptions that are based on the premise of a "lean" approach [18]. Because it is mainly intended for small and medium-sized organisations or enterprises, which as a rule have limited resources, it is oriented towards simplification, transparency and target orientation. Since various models, sub-models, forms, indicators, methods and techniques exist, in the following a description of the model's functioning is given or, based on this, a simulation of change management is performed.

The model in Fig. 1 depicts five basic pillars in blue that (based on selected indicators) lead the user to the end goal - a successful implementation of change with clear and measurable results and effects. The model is supported by software which optimises the implementation of operations and is widely applicable for different target groups. 


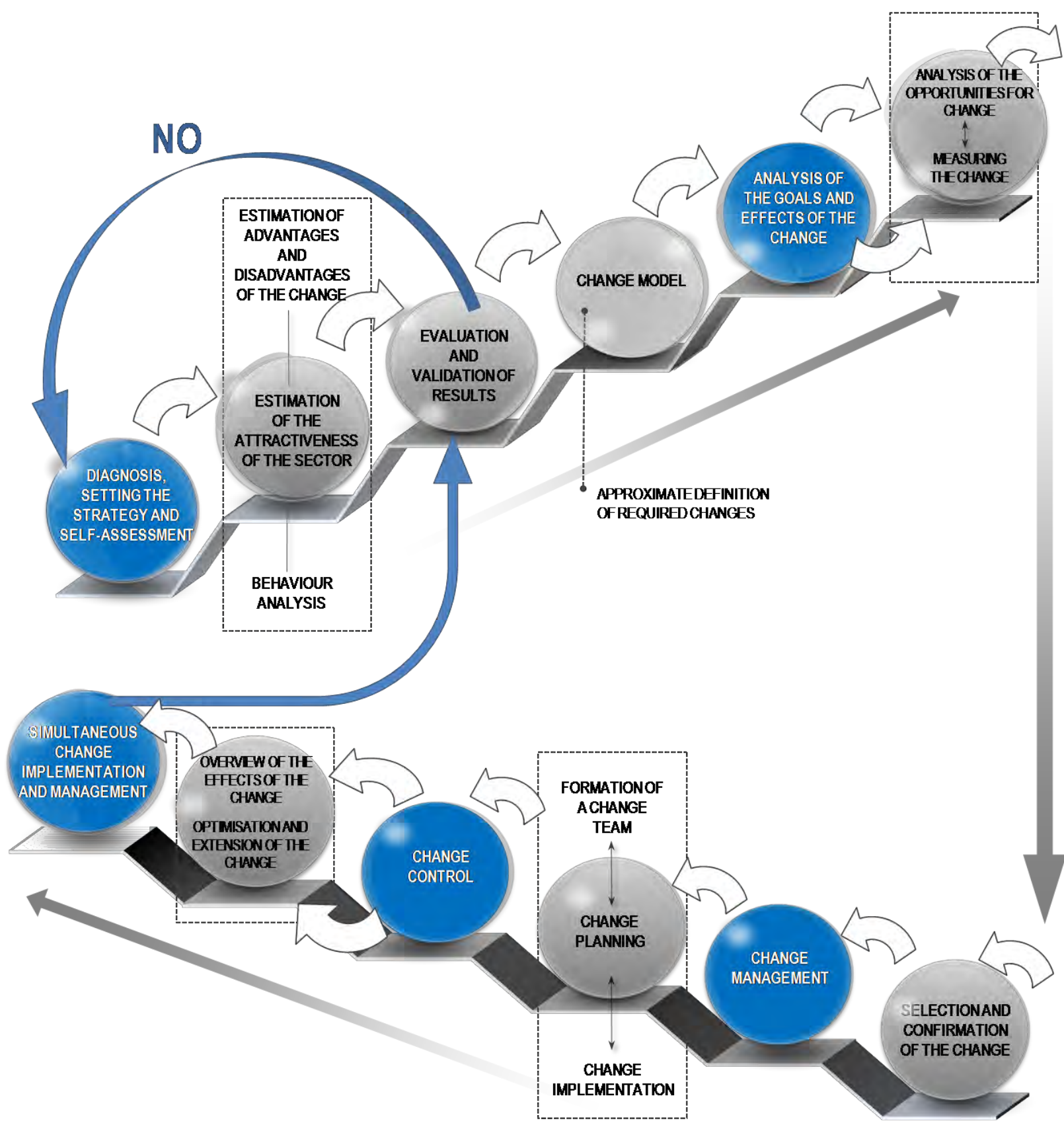

Figure 1: Change management model.

Phase one gives emphasis to the diagnosis, self-assessment of the state, the conditions in an organisation and the broader environment; this is the basis for defining strategic decisions and setting basic guidelines for the activities. The most prevalent aspects of the organisation's life must be defined; on this basis an approach to the change can be identified. Assessment models are defined which as an integral part of the generic model recognise the need for introducing change and trigger the change process. This model includes an assessment of the state of an organisation, as well as a growth forecast in the organisation's broader environment. Different structures and substructures are being assessed which are evaluated through weightings. The model is applicable because it gives an objective assessment of the state of an organisation and its broader environment, based on which a strategy and changes needed for its implementation can be defined. Furthermore, reasons are analysed which identify the phase in the life cycle and lead to the growth and aging of an organisation. If strategic planning goals are set they can be compared with financial results which the organisation would achieve in the years to come if no important changes were made. A simple 
question must be answered: What is the difference between the planned goal and what is happening now? The answer to this question is a certain gap or difference between the results hoped for and the results that would be achieved if no great operational changes were made [19]. This means the goals are being defined and tested while the limitations are considered, and at the same time change measurement is being introduced.

It has been highlighted repeatedly that management is of key importance for implementing changes. But the management can only be successful if the team is good and motivated. The formation of the change team should not be performed arbitrarily; it should follow certain previously defined criteria. Moreover, all activities have to be performed or implemented according to the plan and the strategy defined. An exact timetable for implementing changes has to be drawn up when the unwanted state in a company or organisation is being changed into the desired one based on planned measures. For this purpose a list of measures or interventions which are activated based on recognised indicators has been defined.

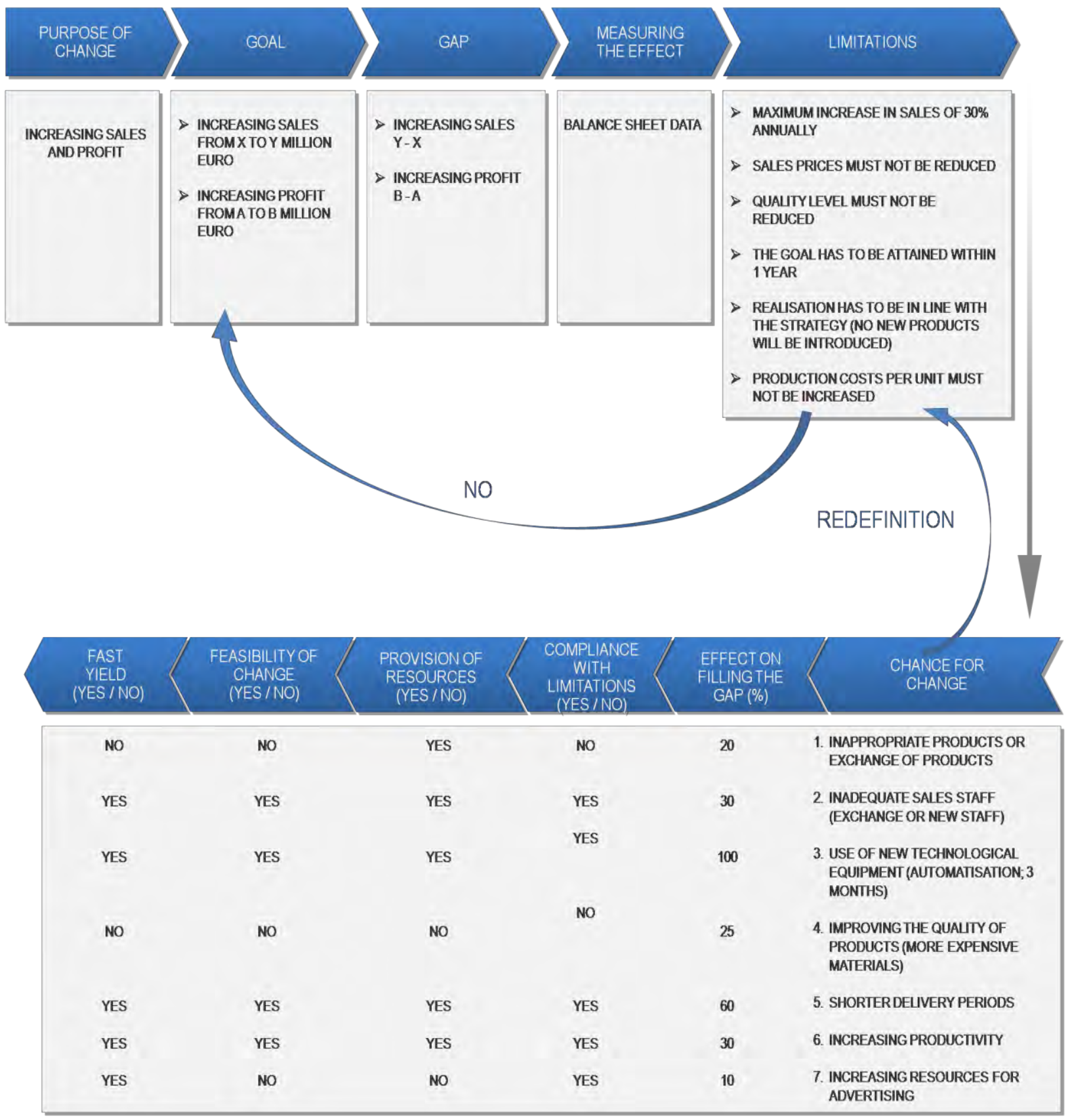

Figure 2: Simulation of a variant solution on the example of increasing sales. 
In the end phase it is of the utmost importance to monitor accurately the change or its effects in order to be able to take measures in due time. Basically, this means returning to measuring or testing the goals, where the measures required are defined based on the results recognised, or the positive effects of change are extended to other company structures. The desired end state is a simultaneous change implementation and management. In this regard the model is a useful tool.

\section{A CHANGE MANAGEMENT SIMULATION BASED ON THE MODEL}

Because this is a broad structure, in the following a simulation with only a few selected parameters is presented or, in other words, the simulated variant in Fig. 2 (see on the previous page) is based only on the example of increased sales.

The purpose of the change is increasing sales, which are defined by a goal. Parallel to the targeted increased sales a targeted increased profit is also planned. The desired state is defined by the gap or by filling this gap. A simple question must be answered: What is the difference between the targeted value and the status quo? It is very important to define how this difference, or effect, will be measured. In this simulation balance sheet data for sales and the profit achieved were used; in general, there are different sources of measurement data which can be used for individual examples. Furthermore, it is advisable to perform an analysis of the impact of the effects and their interaction and an analysis of potential threats.

\section{RESULTS OF THE SIMULATION}

Companies have various goals which they want to attain. Table I shows the most common or desired ones, according to the type of company active on the market. At the same time, a simulation of potential threats is presented which can occur while implementing changes.

Table I shows a simulation of a few possible risks in implementing changes. It is demonstrated that this is a greatly intertwined development, where pursuing certain goals can lead to a total collapse of a stable state in other areas. The realisation of a certain goal can lead to a collapse of all other goals. For example, if the aim is to increase sales at all costs, the sales prices could be reduced and the advertising budget increased; but if this process is performed uncontrolled, the result will be poor in spite of increased sales. The key goal which should be pursued in this case is profitability. Lower costs, higher prices and an increased sales volume are contributing factors to increased profitability, but improving only one of them does not guarantee high profits. It is hard to limit the performed change to only one area. The effect of the change is wider and the presented simulations are just a narrow depiction of a potential course of action. The developed model includes a wide range of different simulations which lead the user to the right decisions, based on entered indicators.

The next simulation phase is the limitations which restrict the goal realisation. For production companies a growth of up to $30 \%$ is recommended. A greater growth could influence the foundation of the company and would therefore represent a risk. In the simulation model the increase in sales is therefore limited to $30 \%$. Other input parameters are prices, quality, costs, new products, time-related targets and strategy. The limitations analysis reveals whether the goal set is feasible or not. If the goal is not feasible, it has to be set once again taking the limitations into consideration. If we set a goal of increasing sales by $40 \%$, this is not in accordance with the limitation that annual growth of more than $30 \%$ is not recommended for production companies. We could still pursue the goal of a $40 \%$ sales increase, but we also have to take appropriate measures to mitigate risks (recapitalisation, strengthening human resources structures, etc.). 
Table I: Simulation of goals of changes as examples and analysis of potential threats.

\begin{tabular}{|c|c|}
\hline Goal of change & Impact of measurements and risks \\
\hline Increasing sales & $\begin{array}{l}\text { The aim is to select changes and activities which are going to increase the sales } \\
\text { volume (increasing production output, which indirectly enables increased sales, } \\
\text { marketing, attractive prices, special offers, new sales staff, etc.). } \\
\text { It is important to be aware of the fact that by only focusing on sales volume the } \\
\text { profitability can decrease, because the goal is only to sell although this may not } \\
\text { be profitable. Many companies experienced a situation where they were only } \\
\text { trying to increase the sales volume at all costs; in the end this led to the } \\
\text { recognition that, although revenues had increased, there were many unprofitable } \\
\text { business partners and programmes which as a whole reduced the profitability of } \\
\text { the company's financial performance. Typical problems connected with this are } \\
\text { complaints and the inability to monitor production quality, because the focus is } \\
\text { only on sales and the company cannot, or does not want to see, these problems. } \\
\text { This can have a negative impact in the years ahead, as unresolved complaints can } \\
\text { have repercussions in the future and may lead to big and sometimes unsolvable } \\
\text { financial consequences. }\end{array}$ \\
\hline Reducing costs & $\begin{array}{l}\text { Activities or changes to reduce costs are performed. The range of such activities } \\
\text { is wide (new technological processes, more efficient processes, reducing wages, } \\
\text { services, complaints, etc.). When performing activities to reduce costs care must } \\
\text { be taken not to miss the opportunity for business growth, as the price for reduced } \\
\text { costs can be a significant decrease in business operations. } \\
\text { If increased productivity and quality are desired as well as reduced production } \\
\text { costs per unit, a prior investment in new equipment is required in the simulated } \\
\text { example. This may be connected to substantial costs in the first phase, but the } \\
\text { change implemented will enable reduced costs in future; this means that the } \\
\text { investment outweighs the costs. Of course the cost rationalisation has to be } \\
\text { carefully monitored and measures have to be taken in due time. The investment } \\
\text { made will enable a larger production output and consequently shorter delivery } \\
\text { periods. } \\
\text { The above-mentioned example demonstrates that the use of new technological } \\
\text { equipment has a great influence on the implementation of other goals and has } \\
\text { therefore a priority role. }\end{array}$ \\
\hline Higher prices & $\begin{array}{l}\text { The aim is to select changes and activities which will increase prices or, in case } \\
\text { of the simulation, maintain the current price level. But although raising prices too } \\
\text { much can still realise a certain amount of sales where the profitability will be } \\
\text { high, there is a risk of losing customers who are willing to pay only the lower } \\
\text { prices. If the additional profit on sales is bigger than the lost sales volume then it } \\
\text { is profitable; otherwise it is not. }\end{array}$ \\
\hline
\end{tabular}

After setting limitations the simulation leads us to the definition of opportunities or possible causes for a gap. Within the framework we defined a database of opportunities, which the user can include into the analysis in specific examples. Every opportunity has to be analysed in terms of effect, limitations, resources provided, fast yield and feasibility.

In the simulated example, opportunities 1 and 4 do not comply with the limitations set; therefore they have to be dismissed. In line with the strategy formed the goal was set not to introduce new products and that at the same time the production costs per unit would not be increased. New products would indeed improve the status quo, and more expensive materials would improve the quality, but the analysed effect is too low in both cases (the gap is insufficiently filled and it takes too long to reach targeted outcomes) in order to be worthwhile redefining the limitations and the goal. 
Opportunity 7 is in line with the limitations; nevertheless, in this case there are insufficient resources provided for implementing activities. The analysed or expected effect of the opportunity is weak as well. Therefore it is estimated that measures for an eventual acquisition of resources are not reasonable.

Based on the value analysis opportunities 2, 3, 5 and 6 are left. All four opportunities enable a fast yield and are feasible, based on the evaluated parameters. Considering the effect it makes sense to first start implementing activity 3 - introducing or using new automated technological equipment which according to plan can be installed and ready for production use within three months. The implementation of this activity directly influences opportunities 5 and 6 , because automated processes are going to increase the productivity, shorten delivery periods and increase profitability. Due to the interconnectedness of opportunities 3,5 and 6 it is reasonable to perform these activities simultaneously. With regard to the interaction of effects an analysis of the impact of effects was also performed. Opportunity 2 or connected activities can be implemented a little later or remain independent of other activities, based on the availability of resources.

The realisation of activities has to fulfil a goal; this is monitored through effect measurement. Activities are performed in accordance with the simulated management model; this means that selected opportunities for change are introduced within the framework of management sub-models and change monitoring.

\section{CONCLUSION}

The purpose or employability of the model developed is that essentially every manager can define and implement the right measures, based on selected indicators and parameters, and create an added value for the company regardless of the company's current state and situation. Basic indicators or parameters are at the user's disposal and simulate typical business events, on which basis the user can identify the state of the company. Emphasis is given to the definition or analysis of the state, the life cycle of the company and the identification, as well as the urgent solving, of pathological problems.

The employability of the model developed is that it changes a static organisation which introduces changes occasionally into a dynamic organisation, capable of fast restructuring when necessary. The model helps to improve existing processes, to introduce new products and technologies; it facilitates further growth and profitability, reduces operational costs, improves the skills and capabilities of employees, adapts organisational structures, improves customer services and brings a new culture into the organisation. The focus is on the concept of a fast presentation of positive results or rapid success; this helps in generating support for a consolidation of change. The model has a medium- and a long-term employability. It enables the company to be prepared at all times or to implement adjustments and a future development more easily. The model treats business operations in a company as an integral whole of processes which act in concert.

It was shown that a number of indicators and parameters exist which affect each other and are interconnected. It is impossible to set the focus only on area in the company, pursue the goal only in this area and then expect to achieve holistic success. The success or failure of a company depends on synchronised activities within the company which react to market demands. The implementation of a change has to be measured in all areas of the company. For this purpose a network of appropriate indicators and parameters has to be set up, and they need to be holistically connected in the measurement process or model. The developed model for managing change includes a sophisticated network of indicators, parameters and processes; nevertheless, the development of such a model is a sustainable process, which in its structure has to consider dynamic movements and changes within and outside the company. 


\section{REFERENCES}

[1] Tajnikar, M. (2000). Risk management: A book about gazelles and growing businesses (in Slovenian), College of Entrepreneurship, Portoroz

[2] Huczynski, A.; Buchanan, D. A. (1985). Organizational Behaviour: An Introductory Text, Longman Higher Education, Boston

[3] Cameron, E.; Green, M. (2011). Making Sense of Change Management: A Complete Guide to the Models, Tools and Techniques of Organizational Change Management $\left(2^{\text {nd }}\right.$ ed.), Kogan Page, London

[4] Paton, R. A.; McCalman, J. (2010). Change Management: A Guide to Effective Implementation $\left(3^{\text {rd }}\right.$ ed.), Sage, London

[5] Psenicny, V. (2002). Conditions and possibilities for dynamic entrepreneurship in Slovenia (in Slovenian), Faculty of Economics, Ljubljana

[6] Mozina, S.; Zorman, R.; Tavcar, M.; Pucko, D.; Kralj, J.; Ivanko, S.; Lipicnik, B.; Gricar, J.; Tekavcic, M.; Dimovski, V.; Kovac, B. (2002). Management: new knowledge for success (in Slovenian), Didakta, Radovljica

[7] Adizes, I. (2006). Mastering Change (in Serbian), Graph Style, Novi Sad

[8] Adizes, I. (2007). Managing Corporate Lifecycle (in Serbian), Asee, Novi Sad

[9] Debevec, M.; Simic, M.; Herakovic, N. (2014). Virtual factory as an advanced approach for production process optimization, International Journal of Simulation Modelling, Vol. 13, No. 1, 66-78, doi:10.2507/IJSIMM13(1)6.260

[10] Stahl, B.; Cerinsek, G.; Colombo, F.; Taisch, M. (2012). Development of competence for sustainable manufacturing by using serious games, Transactions of FAMENA, Vol. 36, No. 4, 6372

[11] Kim, S. T.; Hong, S. R.; Kim, C. O. (2014). Product attribute design using an agent-based simulation of an artificial market, International Journal of Simulation Modelling, Vol. 13, No. 3, 288-299, doi:10.2507/IJSIMM13(3)3.266

[12] Hayes J. (2010). The Theory and Practice of Change Management ( $3^{\text {rd }}$ ed.), Palgrave MacMillan, New York

[13] Abrahamson, E. (2004). Change Without Pain: How Managers Can Overcome Initiative Overload, Organizational Chaos, and Employee Burnout, Harvard Business School Press, Boston

[14] Antariksa, Y. (2007) Change Management: Leading Corporate Transformation, from http://www.slideshare.net/nusantara99/change-management-51890, accessed on 06-07-2014

[15] Ash, P. (2009). Fast and Effective Change Management, Knowledge Solutions, from http://www.adb.org/Documents/Information/Knowledge-Solutions/fast-effective-changemanagement.pdf, accessed on 07-07-2014

[16] Darwin, J.; Johnson, P.; McAuley, J. (2002). Developing Strategies for Change, Prentice Hall, Harlow

[17] Accenture. Change management: Service overview, from http://www.accenture.com/usen/Pages/service-change-management-overview-summary.aspx, accessed on 13-08-2014

[18] Gracanin, D.; Lalic, B.; Beker, I.; Lalic, D.; Buchmeister, B. (2013). Cost-time profile simulation for job shop scheduling decisions, International Journal of Simulation Modelling, Vol. 12, No. 4, 213-224, doi:10.2507/IJSIMM12(4)1.237

[19] Whelan-Berry, K. S.; Gordon, J. R. (2000). Effective organizational change: new insights from multi-level analysis of the organizational change process, Academy of Management Proceedings, D1-D6 